\title{
ELECTRONIC SPECTRA OF 1-PHENYLETHYLAMINE AND ITS DERIVATIVES IN SUPERSONIC JETS
}

\author{
SEIJI YAMAMOTO, TAEKO NIWA AND MITSUO ITO \\ Department of Chemistry, Faculty of Science, \\ Tohoku University, Sendai 980, Japan
}

(Received 20 June, 1989; in final form 19 July 1989)

The $S_{1} \leftarrow S_{0}$ fluorescence excitation spectra and dispersed fluorescence spectra of jet-cooled (+)-, (-)and ( \pm )-1-phenylethylamine and their derivatives (amides) have been observed. The $0-0$ band of the amine locates at $37,641 \mathrm{~cm}^{-1}$. The amides which were synthesized from (+)-amine or (-)-amine with $(+)$-tartaric acid are diastereomers. It was found that the two diastereomers give the identical spectra with the $0-0$ band at $34,757 \mathrm{~cm}^{-1}$. No difference in the spectrum indicates that the excitation is localized in the phenyl group which is far from the asymmetric carbon causing diastereoism. It was also found that 1-phenylethylamine has a fast nonradiative relaxation process in the $S_{1}$ state, but such a process is removed by the formation of the amide.

KEY WORDS: Electronic spectra, supersonic jets, tartaric acid, 1-phenylethylamine, N,N'-di-1phenylethyltartaramide, diastereomers.

\section{INTRODUCTION}

The beginning of optical resolution goes back to the middle of the nineteenth century. Pasteur resolved the crystals of $\mathrm{Na}\left(\mathrm{NH}_{4}\right) \mathrm{C}_{6} \mathrm{H}_{4} \mathrm{O}_{4} \cdot 4 \mathrm{~h}_{2} \mathrm{O}$ by tweezers (mechanical resolution). The most general method of optical resolution is that of changing a racemic form to diastereomers and using the difference in their physical properties. $^{1,2}$ It is interesting to see whether there is appreciable difference between the electronic spectra of such diastereomers. Since the difference is expected to be very small, subtle difference will be found only when the spectrum exhibits a wellresolved structure. The electronic spectrum of a molecule in a supersonic jet generally gives a well-resolved vibrational structure because of elimination of hot bands. Therefore, the supersonic jet electronic spectroscopy might be useful for the discrimination of the diastereomers.

In the present study, we selected optical active (+)- and (-)-1-phenylethylamine (Figure 1) and observed their electronic spectra under supersonic jet conditions. The two spectra were found of course to be completely identical. Diastereomers were prepared by gas-phase reaction of the optical active amines and (+)-tartaric acid (Figure 1) inside a supersonic apparatus at high temperature. The reaction products were the diastereomers of amide, whose electronic spectra were measured under the supersonic jet conditions. 
<smiles>CC(N)c1ccccc1</smiles>

$(+)-$<smiles>CC(N)c1ccccc1</smiles>

$(-)-$

1 - phenylethylamine<smiles>O=C(O)C(O)C(O)C(=O)O</smiles>

(+)-tartaric acid

Figure 1 The molecular structures of $(+)$ - and $(-)-1$-phenylethylamine and (+)-tartaric acid.

\section{EXPERIMENTAL}

The fluorescence excitation and dispersed fluorescence spectra in a supersonic jet were measured with the same apparatus as that reported elsewhere. ${ }^{3}$ The sample was seeded in $\mathrm{He}$ and the gaseous mixture ( $3 \mathrm{~atm})$ was expanded into a vacuum chamber $\left(\sim 10^{-5}\right.$ Torr $)$ through a pulsed nozzle with $0.4 \mathrm{~mm}$ diameter orifice. The repetition frequency was $10 \mathrm{~Hz}$. The fluorescence excitation spectra were obtained by monitoring the total fluorescence from $S_{1}$ with a photomultiplier (Hamamatsu photonics R-562). The photocurrent signal was averaged by a boxcar integrator (Brookdeal 9415/9425) and recorded on a chart recorder. The exciting source was the second harmonic (KDP or $\beta-\mathrm{BaB}_{2} \mathrm{O}_{4}$ ) of a dye laser (Molectron DL-14P) pumped by a nitrogen laser (Molectron UV-24). The fluorescence was dispersed by a Nalumi $0.75 \mathrm{~m}$ grating monochromator in its second order with a $0.1 \mathrm{~mm}$ slit width and was detected by a photomultiplier (Hamamatsu photonics R-928). The signal was processed by the same integrator system as that for the fluorescence excitation spectrum measurement. The samples used were as follows.

$$
\begin{aligned}
& \text { R-(+)-1-phenylethylamine (Tokyo Kasei purity }>99 \% \text { ). } \\
& \text { S-(-)-1-phenylethylamine (Tokyo Kasei purity }>99 \% \text { ). } \\
& \text { R,S-( }( \pm)-1 \text {-phenylethylamine (Tokyo Kasei purity }>99.8 \% \text { ). } \\
& \text { (R,R)-(+)-tartaric acid (Tokyo Kasei purity }>99.8 \% \text { ) }
\end{aligned}
$$

They were used without further purification. For 1-phenylethylamine monomer, the sample was heated up to $300 \mathrm{~K}$ to obtain sufficient vapor pressure. The amide formed between 1-phenylethylamine and tartaric acid was synthesized by heating the gaseous mixture up to about $400 \mathrm{~K}$ in the nozzle. 


\section{RESULTS AND DISCUSSION}

\section{1-phenylethylamine}

Figure 2(a) shows the $S_{1} \leftarrow S_{0}$ absorption spectrum of (+)-1-phenylethylamine vapor at room temperature and Figure 2(b) shows the corresponding fluorescence excitation spectrum of the jet-cooled molecule. The $0-0$ band is at $37,641 \mathrm{~cm}^{-1}$ and the bands involving the $6 \mathrm{~b}$ mode $\left(523 \mathrm{~cm}^{-1}\right.$ in $\left.\mathrm{S}_{1}\right)$ appear strongly in both the absorption and excitation spectra. Therefore the $\mathrm{S}_{1} \leftarrow \mathrm{S}_{0}$ spectrum is benzene like. The fluorescence excitation spectrum of the jet-cooled molecule (Figure 2(b)) exhibits a well-resolved vibrational structure. The $0-0$ band and the band involving one quantum of the $6 \mathrm{~b}$ mode appear in the spectrum with fairly large intensities. However, the intensity suddenly decreases in the frequency region above the excess

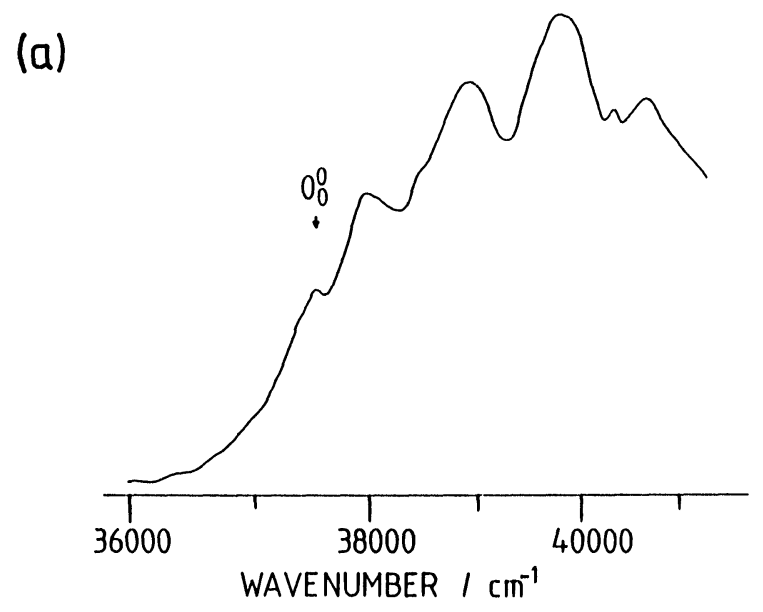

(b)

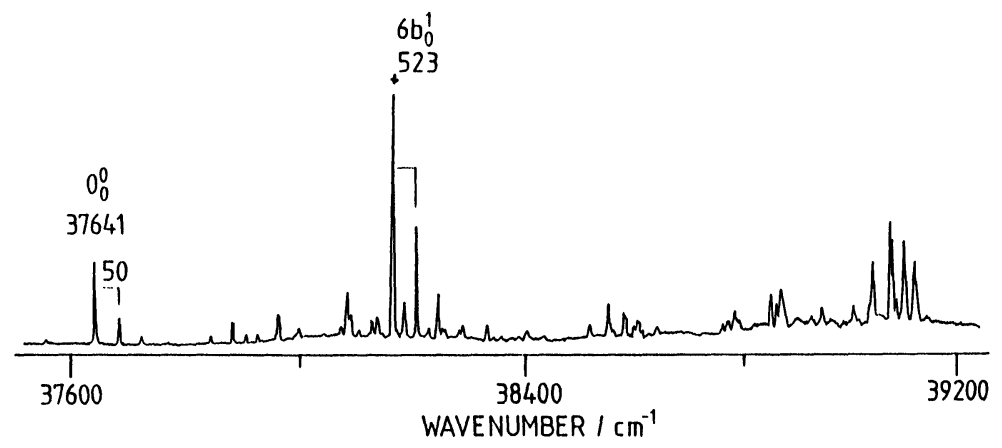

Figure $2 S_{1} \leftarrow S_{0}$ absorption spectrum of (+)-1-phenylethylamine in the vaper phase at room temperature (a) and $S_{1} \leftarrow S_{0}$ fluorescence excitation spectrum of it in a supersonic jet (b). 
energy of about $1500 \mathrm{~cm}^{-1}$ from the $0-0$ band. This exhibits quite a contrast to the vapor absorption spectrum where the bands of the progression of the mode 1 $\left(\sim 900 \mathrm{~cm}^{-1}\right)$ starting from the $6 \mathrm{~b}^{1}$ band appear strongly in the same region. The sudden drop-off of the fluorescence intensity suggests the existence of a fast nonradiative process for the levels above the excess energy of about $1500 \mathrm{~cm}^{-1}$. The low frequency of about $50 \mathrm{~cm}^{-1}$ is seen which is developed from the $0-0$ band and $6 \mathrm{~b}$ bands. This low frequency mode is probably assigned to a torsional mode of the substituent with respect to the phenyl group.

Figure 3 shows the dispersed fluorescence spectra of jet-cooled (+)-1-phenylethylamine obtained by exciting $0-0$ band (a) and $6 b^{1}$ band (b), respectively. The vibrational assignments are shown in the figure. The low frequency progression of 41 $\mathrm{cm}^{-1}$ corresponds to the progression of $50 \mathrm{~cm}^{-1}$ in the fluorescence excitation spectrum. The frequency is larger in the excited state than in the ground state, indicating less flexibility of the tortional motion in the excited state. The characteristic ground-state vibration of amine is found at $587 \mathrm{~cm}^{-1}\left(\gamma_{\mathrm{s}} \mathrm{HN}_{2}\right)$. In the dispersed fluorescence spectrum of the $6 \mathrm{~b}^{1}$ band, the combination bands involving $6 \mathrm{~b}$ are

(a)

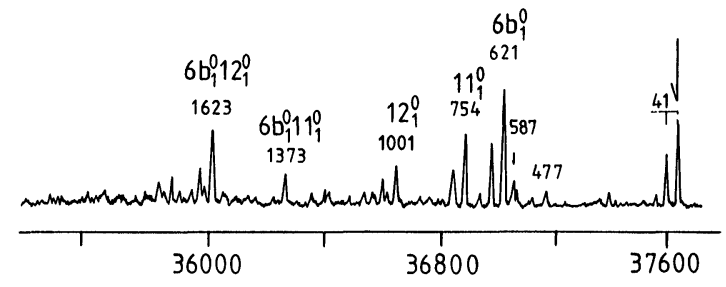

WAVENUMBER $/ \mathrm{cm}^{-1}$

(b)

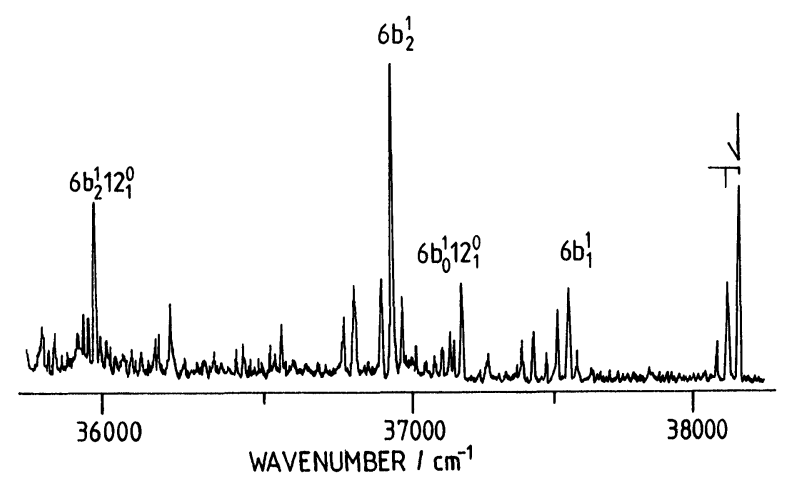

Figure 3 Dispersed fluorescence spectra of (+)-1-phenylethylamine obtained by exciting the 08 band (a) and the $6 b_{0}^{1}$ band (b). Exciting positions are shown by arrows. Vibrational frequencies in the $S_{0}$ state and their assignments are also shown. The band at $587 \mathrm{~cm}^{-1}$ is due to the characteristic vibrational mode of $\mathrm{NH}_{2}$ group. 
Table 1 Correspondence between vibrational frequencies in ground and excited states of 1 phenylethylamine

\begin{tabular}{lccc}
\hline & \multicolumn{2}{c}{ Vibrational frequency $\left(\mathrm{cm}^{-1}\right)$} \\
\cline { 2 - 4 } Mode $^{\mathrm{a}}$ & IR, Raman & Ground state & Excited state \\
\hline 6a & 482 & 477 & 452 \\
6b & 619 & 621 & 523 \\
11 & 768 & 754 & $923 ?$ \\
12 & 1001 & 1001 & \\
\hline
\end{tabular}

Mode number was taken from ref. 4.

dominant, particularly the overtone of $6 \mathrm{~b}$ being the strongest. The ground-state vibrational frequencies obtained from the dispersed fluorescence spectra are in good agreement with those of IR and Raman. ${ }^{4}$ Some of them are listed in Table 1, together with the excited-state frequencies.

Corresponding spectra were also observed for (-)-1-phenylethylamine and (士)-1-phenylethylamine in supersonic jets. The spectra were of course identical with those of $(+)-1$-phenylethylamine.

\section{2. $N, N^{\prime}$-di-1-Phenylethyltartaramide}

1-phenylethylamine vapor and solid tartaric acid forms a salt at room temperature. It is known that the salt obtained from (-)-amine and (+)-acid is formed more easily than that from $(+)$-amine and $(+)$-acid. ${ }^{5}$ By using this difference, the resolution of (士)-1-phenylethylamine was performed. ${ }^{1}$ It is also known that the salt reacts to form an amide with a very high yield at high temperature as follows.

$$
-\mathrm{COOH}+-\mathrm{NH}_{2} \stackrel{\Delta}{\longrightarrow}-\mathrm{CONH}+\mathrm{H}_{2} \mathrm{O}
$$

In tartaric acid there are two functional groups of $\mathrm{COOH}$. Under the presence of the abundant amine which is realized in the nozzle chamber at high temperature (400 $\mathrm{K})$, the acid and the amine are thought to react at the ratio of $1: 2^{5}$. The formation of the amide was confirmed by micro analysis and also by NMR and IR spectra. The two amides (N,N'-di-1-phenylethyltartaramides formed from the (+)- and (-)-amines) are diastereomers as shown in Figure 4.

\section{diastereomers}
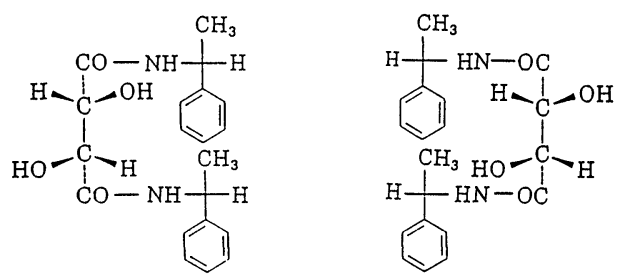

$N, N^{\prime}$-di-1-phenylethyltartaramide
Figure 4 The molecular structures of the diastereomers of $\mathbf{N}, \mathbf{N}^{\prime}$-di-1-phenylethyltartaramide formed from $(+)$ - or $(-)-1$-phenylethylamine and $(+)$-tartaric acid. 
Figure 5 shows the $S_{1} \leftarrow S_{0}$ fluorescence excitation spectrum of jet-cooled N,N'-di-1-phenylethyltartaramide prepared from a (+)-1-phenylethylamine and $(+)$-tartaric acid. Since the spectrum was found to be completely the same as that of the amide prepared from (-)-1-phenylethylamine and (+)-tartaric acid, we do not specify which amide in the description of the spectrum. The spectrum exhibits a well-resolved vibrational structure over a wide spectral region. The longest wavelength band at $34,757 \mathrm{~cm}^{-1}$ is assigned to the $0-0$ band. The vibrational structure is developed from this $0-0$ band until the excess energy more than $2800 \mathrm{~cm}^{-1}$, where several sharp bands due to nonreactive amine are seen. Therefore, the electronic state of the amide is greatly stabilized by $2800 \mathrm{~cm}^{-1}$ compared with that of the amine. The well-resolved vibrational structure in the fluorescence excitation spectrum over a wide spectral region is in contrast to the sharp drop-off of the fluorescence intensity of the amine at the excess energy above about $1500 \mathrm{~cm}^{-1}$. Therefore, the nonradiative decay process existing in the amine is eliminated by the formation of the amide. This suggests that the flexible $\mathrm{NH}_{2}$ group involves in the nonradiative process which is removed by fixing the group by the formation of the amide. Although the vibrational structure of the fluorescence excitation spectrum looks complex, it can be analyzed as repeated appearance of a low-frequency structural unit in combination with the strong main vibronic bands involving the excited-state fundamentals as shown in the figure. The structural unit consists of $236,283,350,372$ and $395 \mathrm{~cm}^{-1}$, which are probably assigned to excited-state vibrations mainly localized near the amido group. The main vibronic bands at 523,746 and $958 \mathrm{~cm}^{-1}$ above the $0-0$ band are assigned to the excited-state fundamentals of the $6 \mathrm{~b}, 11$ and 12 modes of phenyl group. The band at $1209 \mathrm{~cm}^{-1}$ is probably assigned to the characteristic vibrational of amide, so called, amide III.

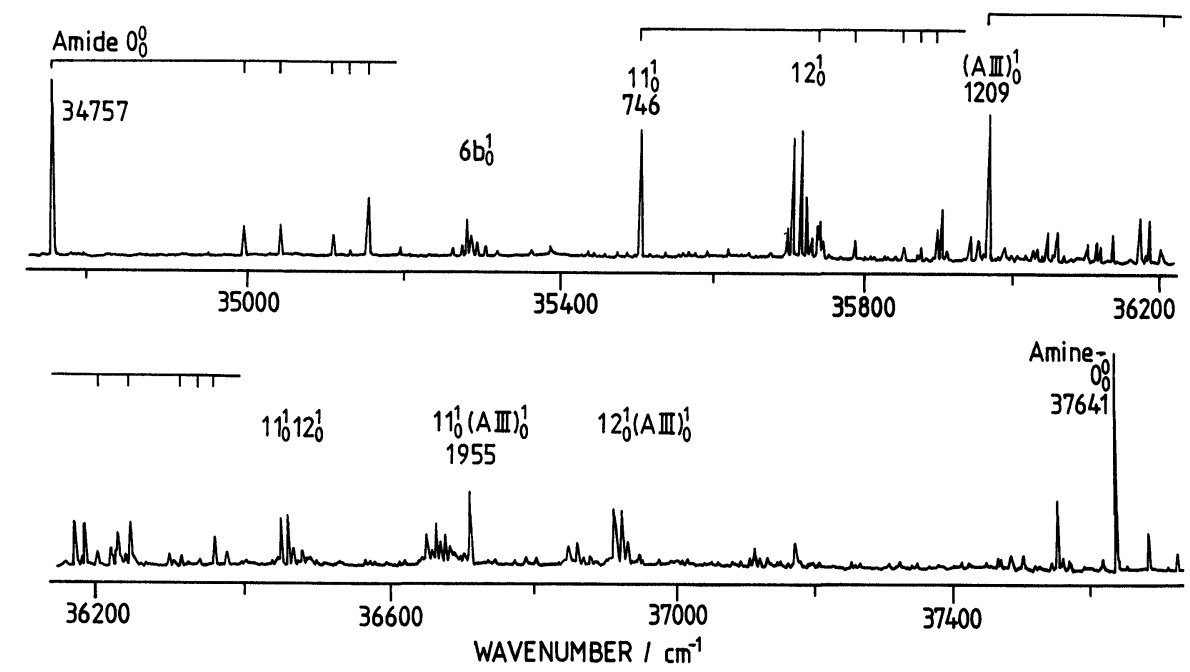

Figure $5 \quad S_{1} \rightarrow S_{0}$ fluorescence excitation spectrum of jet-cooled $N, N^{\prime}$-di-1-phenylethyltartaramide. The 08 band of the amide is located at $34,757 \mathrm{~cm}^{-1}$. A few assignments are also shown. AIII denotes the vibrational mode of amide III. 


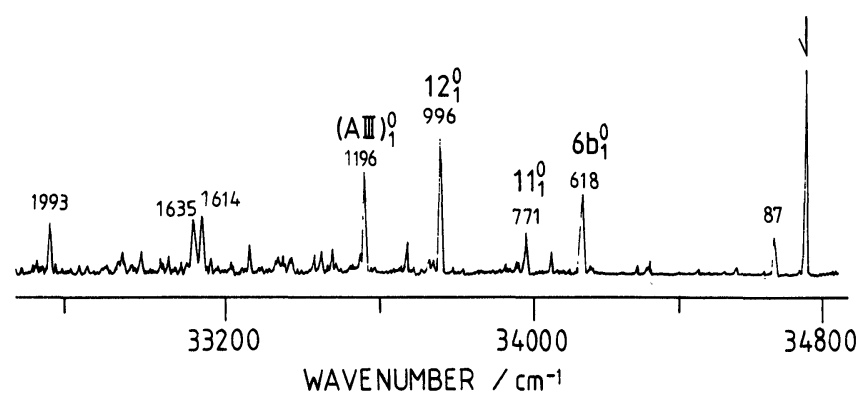

Figure 6 Dispersed fluoresence excitation spectrum of N,N'-di-1-phenylethyltartaramide obtained by exciting the 08 band. Excitation position is shown by an arrow. A few assignments are also shown. AIII denotes the vibrational mode of amide III.

Figure 6 shows the dispersed fluorescence spectrum obtained by exciting the $0-0$ band. The structure of the spectrum shows a good mirror image to that of the fluorescence excitation spectrum. From this good mirror image, we can immediately obtain the ground-state vibrational frequencies of $6 \mathrm{~b}, 11$ and 12 and amide III, which are $618,771,996$ and $1196 \mathrm{~cm}^{-1}$. In general, the vibrational frequencies of the amide are similar to those of amine for both the ground and excited states. This indicates that the nature of the electronic transition of the amide is essentially the same as that of the amine and the part of the tartaric acid in the amide is not directly involved in the electronic transition.

As seen from Figure 4, the asymmetric carbon atom in the tartaric acid part produces diastereoism. However, it is located far from the phenyl group whose $\pi$ electron is mainly involved in the electronic excitation. Therefore, the difference in the geometrical structure of the tartaric acid part does not cause an appreciable difference in the spectrum between the two amides prepared from (+)- and (-)amines. This is probably the reason for the failure of the discrimination of the diastereomers by their electronic spectra. A large difference will be found if we select the electronic transition which is more closely associated with the part of tartaric acid.

\section{Acknowledgements}

We thank Dr Y. Morioka for taking IR spectra and Mr T. Kondow for taking NMR spectra. We also thank Drs N. Mikami, T. Ebata and M. Fujii for helpful simultaneous discussions.

\section{References}

1. A. Ault, Org. Syn. Coll. Vol. V. 932 (1973).

2. H. Nohira, M. Kai, M. Nohira, J. Nishikawa, T. Hoshiko and K. Saigo, Chem. Lett. 951 (1981).

3. N. Mikami, A. Hiraya, I. Fujiwara and M. Ito, Chem. Phys. Lett. 74, 531 (1980).

4. G. Varanze, "Assignment for Vibrational Spectra of Seven Hundred Benzen Derivatives," Adam Hilger, London, 1974.

5. C. T. Lin, D. Y. Curton and I. C. Paul, J. Am. Chem. Soc. 96, 6199 (1974). 